\title{
ANALYTICAL MODELING OF DISCRIMINATION SCHEME FOR DETECTION OF ANGULAR RATE AND ACCELERATION FOR A 4-DOF MEMS GYRO- ACCELEROMETER
}

\author{
Payal Verma, S.A. Fomchenkov \\ Samara National Research University, Samara, Russia
}

\begin{abstract}
The paper reports an analytical model of a 4-DOF gyroaccelerometer consisting of 2-DOF drive and 2-DOF sense oscillators configured orthogonally. A detection scheme for time varying angular rate and linear acceleration, by combining the structural-model of gyro-accelerometer with the processes of synchronous demodulation and filtration, which leads to the inphase and quadrature components of the system's output signal. These two components can be utilized in the detection of angular motion and linear acceleration. The in-phase signal can be used for angular rate detection and the quadrature signal can be utilized for linear acceleration. Finally, the results of the model have been validated by comparing with MATLAB ${ }^{\circledR} /$ Simulink data which shows excellent matching with each other.
\end{abstract}

Keywords: MEMS, 4-DOF, Gyro-accelerometer, Synchronous demodulation

Citation: Verma Payal, Fomchenkov SA. Analytical modeling of discrimination scheme for detection of angular rate and acceleration for a 4-DOF MEMS gyro-accelerometer. CEUR Workshop Proceedings, 2016; 1638: 700-708. DOI: 10.18287/1613-0073-2016-1638-700-708

\section{Introduction}

It is a well-known fact that all vibratory gyroscopes operate on the basis of transfer of energy from one mode to the other. The device may have either single DOF [1] or multi-DOF oscillators [2-5] which act as two orthogonally configured subsystems, a self-tuned oscillator forming the drive mode and a micro-g accelerometer, forming the sense mode. In the event of an angular rate, the transfer of energy from one mode to the other is detected and processed using suitable circuitry to produce the desired output. It is quite obvious that all vibratory gyroscopes can also sense linear acceleration in addition to angular rate sensing at their events of occurrence. Considering the strategy of simultaneous detection of linear acceleration and angular rate at their events, a controller circuit has been reported for a 2-DOF conventional gyroscope [6]. 
Some multi-DOF systems have also been proposed and realized which can sense linear acceleration along with angular rate [7,8], while offering other advantages such as increased robustness and immunity to fabrication imperfections.

For the development of superior performance inertial sensors, the characteristics of the device have to be thoroughly understood and the design optimized which can achieved by taking proper care in the design and modeling stages. Hence mathematical modeling plays a key role in device design as well for optical elements [9-13]. Various mathematical models have been reported separately for accelerometer and gyroscope devices. Some of the mathematical models for gyroscopes have reported acceleration effect as an error, however confirming its presence. Recently, mathematical models of multi-DOF structures for simultaneous detection of acceleration effect and angular rate have also been reported, few of them are, 2-DOF gyro-accelerometer [14], a 2-DOF drive and 1-DOF sense gyro-accelerometer [7, 15, 16] and a 1-DOF drive and 2-DOF sense gyro-accelerometer [8].

Drive mass $\left(m_{1}\right)$

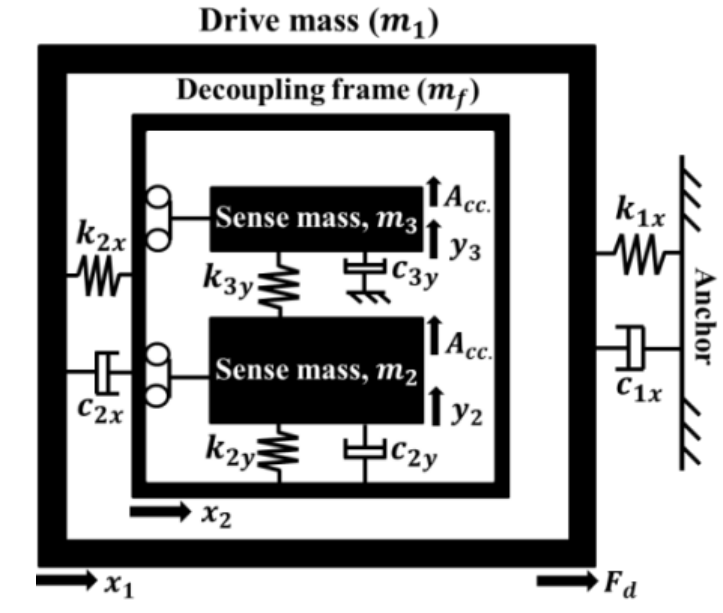

Fig. 1. Schematic illustration of mass-spring-damper for a 4-DOF gyro-accelerometer

\section{$2 \quad$ 4-DOF Model}

The 4-DOF gyro-accelerometer system exploits the dynamic amplification in the decoupled 2-DOF drive and sense oscillators so as to attain large amplitude of oscillation without resonance [17]. As shown in Fig. 1, this system is composed of three proof masses that are interconnected by flexures springs. The mass $m_{1}$ is designed such that it is constrained in the sense direction whilst it is excited in the drive direction alone. The configuration of the masses $m_{2}$ and $m_{3}$ within the decoupling frame mass, $m_{f}$ is such that these two masses are mechanically decoupled from the drive oscillations. Therefore, the first mass $m_{1}$ and the combination of mass $m_{2}$, mass $m_{3}$ and the decoupling frame mass $m_{f}$ i.e. $\left(m_{2}+m_{3}+m_{f}\right)$ form the 2-DOF oscillator in the drive direction. The masses $m_{2}$ and $m_{3}$ oscillate independantly in the sense direction thus forming the 2-DOF sense direction oscillator. The larger mass $m_{2}$ represents the primary mass, which generates the Coriolis force that excites the sense oscillator. 
The smaller mass $m_{3}$ is a secondary mass and is designed to serve as a dynamic vibration absorber of mass $m_{2}$ in the sense direction. In the event of an angular motion, the sense masses $m_{2}$ and $m_{3}$ are influenced by Coriolis force and begin to oscillate in the sense direction. Each of both 2-DOF drive and sense oscillators has two resonance peaks and flat zone in between peaks. The most important requirement of the overall 4-DOF gyro-accelerometer system is that the flat amplitude regions of both the 2DOF oscillators must overlap precisely and the operating frequencies of the system must be located in their flat amplitude zones, thereby leading to the maximum robustness of the performance against the fluctuations of system parameters. The equations of motion can be represented by Newton's second law of motion, [2, 17]:

$m_{1} \ddot{x}_{1}+c_{1 x} \dot{x}_{1}+\left(k_{1 x}+k_{2 x}\right) x_{1}=k_{2 x} x_{2}+F_{d}(t)$,

$M_{p} \ddot{x}_{2}+c_{2 x} \dot{x}_{2}+k_{2 x} x_{2}=k_{2 x} x_{1}$,

$m_{2} \ddot{y}_{2}+c_{2 y} \dot{y}_{2}+\left(k_{2 y}+k_{3 y}\right) y_{2}=k_{3 y} y_{3}-2 m_{2} \Omega_{z} \dot{x}_{2}$

$-m_{2} \dot{\Omega}_{z} x_{2}+m_{2}\left(a_{x} \sin \theta-a_{y} \cos \theta\right)$,

$m_{3} \ddot{y}_{3}+c_{3 y} \dot{y}_{3}+k_{3 y} y_{3}=k_{3 y} y_{2}-2 m_{3} \Omega_{z} \dot{x}_{2}$

$-m_{3} \dot{\Omega}_{z} x_{2}+m_{3}\left(a_{x} \sin \theta-a_{y} \cos \theta\right)$,

respectively where, $F_{d}(t)=F_{o} \sin \omega_{d} t$ is the sinusoidal driving force that excites the driven mass $m_{1}$ at the drive frequency $\omega_{d}, \Omega_{z}=\Omega_{o} \cos (\alpha t)$, where $\Omega_{o}$ is the amplitude of angular velocity, $\alpha$ is the frequency of angular rate. $c_{1 x}, c_{2 x}, c_{2 y}$ and $c_{3 y}$ are the damping coefficients corresponding to the respective stiffness co-efficients $k_{1 x}$, $k_{2 x}, k_{2 y}$ and $k_{3 y}$ as shown in Fig. 1 . The term $\dot{\Omega}_{o} x_{2}$ is the Euler's acceleration. The notations $a_{x}$ and $a_{y}$ are external accelerations along their respective axes and these have been included for the analysis of gyro-accelerometer system. The terms $2 m_{2} \Omega_{o} \dot{x}_{2}$ and $2 m_{3} \Omega_{o} \dot{x}_{2}$ are the Coriolis forces that excite the respective masses, $m_{2}$ and $m_{3}$ in sense direction. The corresponding displacements of the masses, $m_{2}$ and $m_{3}$, are also affected by external linear acceleration.

\section{Detection Scheme}

The scheme for the discrimination of the angular rate and acceleration is presented below. This scheme for angular rate amplitude, $\Omega_{o}$, and associated frequency, $\alpha$, along with linear acceleration is applicable in case where angular rate is time dependent. The absolute transformation of (4) yields the solution that comprises both temporally damped and un-damped terms. The temporal decay terms, however, are not trivial as these are vital for deciding the turn-on time and settling time of the system. Since the output signal is processed after the device output is settled down, the contributions of decay terms become insignificant. Therefore the settled solution, of (4), is written as:

$$
\begin{aligned}
& \bar{y}_{3}(t)=A_{1} \cos \left\{(\omega+\alpha) t+\phi_{c x}(\omega)+\phi_{2 y}(\omega+\alpha)+\phi_{c y}(\omega+\alpha)\right\} \\
& +A_{2} \cos \left\{(\omega-\alpha) t+\phi_{c x}(\omega)+\phi_{2 y}(\omega-\alpha)+\phi_{c y}(\omega-\alpha)\right\} \\
& +\mathcal{R}_{e x} \mathcal{A}_{2 y}(\omega) \mathcal{A}_{c y}(\omega) \sin \left(\omega t+\phi_{2 y}(\omega)+\phi_{c y}(\omega)\right), \\
& \text { where } \mathcal{R}_{e x}, \text { external acceleration and, }
\end{aligned}
$$




$$
\begin{aligned}
& A_{1,2}=-\Omega_{0} f_{o} \omega_{2 x}^{2} \mathcal{A}_{c x}(\omega)\left(\omega \pm \frac{1}{2} \alpha\right) \mathcal{A}_{2 y}(\omega \pm \alpha) \mathcal{A}_{c y}(\omega \pm \alpha), \\
& \mathcal{A}_{c x}^{-2}(\omega)=\left[\left(\omega_{1 x}^{2}-\omega^{2}\right)\left(\omega_{2 x}^{2}-\omega^{2}\right)-\mu_{x}^{2} \omega_{2 x}^{4}-4 \lambda_{1 x} \lambda_{2 x} \omega^{2}\right]^{2} \\
& +4\left[\lambda_{1 x}\left(\omega_{2 x}^{2}-\omega^{2}\right) \omega+\lambda_{2 x}\left(\omega_{1 x}^{2}-\omega^{2}\right) \omega\right]^{2}, \\
& \phi_{c x}(\omega)=-\tan ^{-1} \frac{2 \omega\left\{\lambda_{1 x}\left(\omega_{2 x}^{2}-\omega^{2}\right)+\lambda_{2 x}\left(\omega_{1 x}^{2}-\omega^{2}\right)\right\}}{\left(\omega_{1 x}^{2}-\omega^{2}\right)\left(\omega_{2 x}^{2}-\omega^{2}\right)-\mu_{x}^{2} \omega_{2 x}^{4}-4 \lambda_{1 x} \lambda_{2 x} \omega^{2}}, \\
& \mathcal{A}_{c y}^{-2}(\omega)=\left[\left(\omega_{2 y}^{2}-\omega^{2}\right)\left(\omega_{3 y}^{2}-\omega^{2}\right)-\mu_{y}^{2} \omega_{3 y}^{4}-4 \lambda_{2 y} \lambda_{3 y} \omega^{2}\right]^{2} \\
& +4\left[\lambda_{2 y}\left(\omega_{3 y}^{2}-\omega^{2}\right) \omega+\lambda_{3 y}\left(\omega_{2 y}^{2}-\omega^{2}\right) \omega\right]^{2}, \\
& \phi_{c y}(\omega)=-\tan ^{-1} \frac{2 \omega\left\{\lambda_{2 y}\left(\omega_{3 y}^{2}-\omega^{2}\right)+\lambda_{3 y}\left(\omega_{2 y}^{2}-\omega^{2}\right)\right\}}{\left(\omega_{2 y}^{2}-\omega^{2}\right)\left(\omega_{3 y}^{2}-\omega^{2}\right)-\mu_{y}^{2} \omega_{3 y}^{4}-4 \lambda_{2 y} \lambda_{3 y} \omega^{2}}, \\
& \mathcal{A}_{2 y}^{2}(\omega)=\left[\left(\omega_{2 y}^{2}+\omega_{3 y}^{2}-\omega^{2}\right)^{2}+4 \lambda_{2 y}^{2} \omega^{2}\right], \\
& \phi_{2 y}(\omega)=\tan ^{-1} \frac{2 \lambda_{2 y} \omega}{\omega_{2 y}^{2}+\omega_{3 y}^{2}-\omega^{2}}, \\
& \omega_{1 x}^{2}=\left(k_{1 x}+k_{2 x}\right) / m_{1} ; \omega_{2 x}^{2}=k_{2 x} / M_{p}, \\
& \omega_{2 y}^{2}=\left(k_{2 y}+k_{3 y}\right) / m_{2} ; \omega_{3 y}^{2}=k_{3 y} / m_{3}, \\
& \mu_{x}^{2} \omega_{2 x}^{2}=k_{2 x} / m_{1} ; \mu_{y}^{2} \omega_{3 y}^{2}=k_{3 y} / m_{2} ; \mu_{x}^{2}=M_{p} / m_{1}, \\
& \mu_{y}^{2}=m_{3} / m_{2} ; f_{o}=F_{o} / m_{1} ; \lambda_{1 x}=c_{1 x} / 2 m_{1}, \\
& \lambda_{2 x}=c_{2 x} / 2 M_{p} ; \lambda_{2 y}=c_{2 y} / 2 m_{2} ; \lambda_{3 y}=c_{3 y} / 2 m_{3} .
\end{aligned}
$$

As is evident from (5), the output signal is modulated by a sinusoidal function. The synchronous demodulation of this signal yields the in-phase and quadrature components defined as $\bar{y}_{p}=\bar{y}_{3}(t) \cos (\omega t)$ and $\bar{y}_{q}=\bar{y}_{3}(t) \sin (\omega t)$ respectively. In order to arrive at the low-pass-filtered solution after demodulation, we primarily deal with the in-phase component, $\bar{y}_{p}$. The quadrature component, $\bar{y}_{q}$, can be tackled accordingly. With the aid of trigonometric identities and settled solution (5), the in-phase component $\bar{y}_{p}$ is rearranged as,

$$
\begin{aligned}
& \bar{y}_{p}=\bar{A}\left\{\cos \left(2 \omega t+\phi_{c x}(\omega)+\bar{\phi}\right)+\cos \left(\phi_{c x}(\omega)+\bar{\phi}\right)\right\} \cos (\alpha t+\Delta \phi) \\
& -\delta A\left\{\sin \left(2 \omega t+\phi_{c x}(\omega)+\bar{\phi}\right)+\sin \left(\phi_{c x}(\omega)+\bar{\phi}\right)\right\} \sin (\alpha t+\Delta \phi) \\
& +\frac{1}{2} \mathcal{R}_{e x} \mathcal{A}_{2 y}(\omega) \mathcal{A}_{c y}(\omega)\left\{\sin \left(\phi_{2 y}(\omega)+\phi_{c y}(\omega)\right)\right. \\
& \left.+\sin \left(2 \omega t+\phi_{2 y}(\omega)+\phi_{c y}(\omega)\right)\right\} .
\end{aligned}
$$

The modified parameters included in (6), as per [6, 7], are defined as,

$$
\begin{aligned}
& \bar{A}=\frac{1}{2}\left(A_{1}+A_{2}\right) ; \delta A=\frac{1}{2}\left(A_{1}-A_{2}\right), \\
& \bar{\phi}=\frac{1}{2}\left[\phi_{2 y}(\omega+\alpha)+\phi_{2 y}(\omega-\alpha)+\phi_{c y}(\omega+\alpha)+\phi_{c y}(\omega-\alpha)\right], \\
& \Delta \phi=\frac{1}{2}\left[\phi_{2 y}(\omega+\alpha)-\phi_{2 y}(\omega-\alpha)+\phi_{c y}(\omega+\alpha)-\phi_{c y}(\omega-\alpha)\right] .
\end{aligned}
$$

Further, the output signals terms $\cos (\alpha t+\Delta \phi)$ and $\sin (\alpha t+\Delta \phi)$ in (6) led by phase shift, $\Delta \phi$, are related to the angular rate. The phase shift, $\Delta \phi$, in these function is 
distorted by frequency, $\alpha$. Hence, it is essential to employ low-pass-filtering in order to eliminate the terms having doubled frequency. Thus, the filtered solution after trigonometric manipulation is given by,

$\bar{y}_{l p}=A_{p} \cos \left(\alpha t+\psi_{p}\right)+\frac{1}{2} \mathcal{R}_{e x} \mathcal{A}_{2 y}(\omega) \mathcal{A}_{c y}(\omega) \sin \left(\phi_{2 y}(\omega)+\phi_{c y}(\omega)\right)$,

where

$A_{p}^{2}=\bar{A}^{2} \cos ^{2}\left(\bar{\phi}+\phi_{c x}(\omega)\right)+\delta A^{2} \sin ^{2}\left(\bar{\phi}+\phi_{c x}(\omega)\right)$,

$\psi_{p}=\Delta \phi+\Delta \varphi_{p}$

$\Delta \varphi_{p}=\tan ^{-1}\left[\frac{\delta A}{\bar{A}} \tan \left(\bar{\phi}+\phi_{c x}(\omega)\right)\right]$

Similarly, the low-pass-filtered quadrature component after demodulation is written as,

$\bar{y}_{l q}=A_{q} \cos \left(\alpha t+\psi_{q}\right)+\frac{1}{2} \mathcal{R}_{e x} \mathcal{A}_{2 y}(\omega) \mathcal{A}_{c y}(\omega) \cos \left(\phi_{2 y}(\omega)+\phi_{c y}(\omega)\right)$,

where

$A_{q}^{2}=\bar{A}^{2} \sin ^{2}\left(\bar{\phi}+\phi_{c x}(\omega)\right)+\delta A^{2} \cos ^{2}\left(\bar{\phi}+\phi_{c x}(\omega)\right)$,

$\psi_{q}=\Delta \phi+\Delta \varphi_{q}$,

$\Delta \varphi_{q}=-\tan ^{-1}\left[\frac{\delta A}{\bar{A}} \cot \left(\bar{\phi}+\phi_{c x}(\omega)\right)\right]$.

It is worth mentioning here that the first terms pertaining to gyro action of the of respective in-phase and quadrature signals (7) and (8) are distorted both in amplitudes, $A_{p}$ and $A_{q}$, and in corresponding phases, $\psi_{p}$ and $\psi_{q}$, by $\alpha$. At the same time, the linear acceleration terms in these signals are unaffected by $\alpha$. From equations (7) and (8) it is also inferred that at the optimum operating condition of the present device, the system must be driven at zero-phase frequency, as already emphasized [17], of the 2DOF drive oscillator and the corresponding structural frequencies, $\omega_{1 x}$ and $\omega_{2 x}$ must be equal and these must match with driving frequency that results into $\phi_{c x}(\omega)=0$. Likewise, the anti-resonance frequency of passive mass $\left(m_{2}\right)$ amplitude must also match with drive frequency and sense mass related structural frequencies, $\omega_{2 y}$ and $\omega_{3 y} \sqrt{ }\left(1+\mu^{2}\right)$, must be equal [17]. That leads to the phase, $\phi_{2 y}(\omega)+\phi_{c y}(\omega)=0$. As a consequence of this the acceleration term in in-phase signal (7) vanishes and only the gyro action related term exists. On the other hand in quadrature signal (8), the acceleration term exist and gyro related term is almost insignificant. Therefore, the in-phase signal (7) can be used for angular rate detection and the quadrature one (8) can be utilized for linear acceleration extraction under such optimized operating condition of the gyro-accelerometer system.

\section{$4 \quad$ Results and discussion}

Considering the design equations, the spring constants and structural frequencies by adjusting mass values, $m_{1}, m_{2}, m_{3}$, frame mass, $m_{f}$ and subsequently mass ratios, $\mu_{x}^{2}$ and $\mu_{y}^{2}$, have been decided optimally. The values of these and other parameters are listed in Table 1. The following figures have been calculated by using these values unless it is specified. 
Table 1. Parameter values used for calculations

\begin{tabular}{ll}
\hline Parameters & Values \\
\hline Active mass $\left(\boldsymbol{m}_{\boldsymbol{1}}\right)$ & $201.9 \times 10^{-9} \mathrm{~kg}$ \\
Passive mass $\left(\boldsymbol{m}_{2}\right)$ & $57.24 \times 10^{-9} \mathrm{~kg}$ \\
Sense mass $\left(\boldsymbol{m}_{\boldsymbol{3}}\right)$ & $5.6 \times 10^{-9} \mathrm{~kg}$ \\
Frame mass $\left(\boldsymbol{m}_{\boldsymbol{f}}\right)$ & $10.5 \times 10^{-9} \mathrm{~kg}$ \\
Spring constant $\left(\boldsymbol{k}_{\boldsymbol{l}} ; \boldsymbol{k}_{2 \boldsymbol{x}}\right)$ & $153.5 \mathrm{~N} / \mathrm{m} ; 87.59 \mathrm{~N} / \mathrm{m}$ \\
Spring constant $\left(\boldsymbol{k}_{2 \boldsymbol{v}} ; \boldsymbol{k}_{3 v}\right)$ & $62.26 \mathrm{~N} / \mathrm{m} ; 6.1 \mathrm{~N} / \mathrm{m}$ \\
Frequencies $\left(\boldsymbol{\omega}_{1 x}=\boldsymbol{\omega}_{2 \boldsymbol{x}}=\boldsymbol{\omega}_{2 \boldsymbol{y}}\right)$ & $5.5 \mathrm{kHz}$ \\
Frequency $\left(\boldsymbol{\omega}_{3 y}\right)$ & $5.25 \mathrm{kHz}$ \\
$\boldsymbol{F}_{\boldsymbol{o}} ;$ Angular rate $\left(\boldsymbol{\Omega}_{\boldsymbol{o}}\right)$ & $2.171 \times 10^{-5} \mathrm{~N} ; 200 \mathrm{rad}$ \\
\hline
\end{tabular}

Figure 2a-d are Bode plots of the demodulated and low-pass-filtered in-phase and quadrature components of amplitudes and corresponding emerged phases of Coriolis and Euler's signal of gyro-accelerometer for different values of driving frequency. Figure 2a illustrates the results of respective in-phase and quadrature components, $A_{p}$ and $A_{q}$, calculated with the help of (7a) and (8a) respectively for driving frequencies, $\omega=\omega_{d}=\omega_{1 x}$ and $\omega_{d}=1.005 \omega_{1 x}$. From this figure it is observed that a slight deviation of driving frequency, $\omega_{d}$, from $\omega_{1 x}$ within the range of drive and sense bandwidth does not have the considerable effect on the components $A_{p}$ and $A_{q}$. For both values of drive frequency, $\omega_{d}$, the in-phase amplitude $A_{p}$ is the major component and increases with $\alpha$ in both the cases. At the same time the quadrature component, $A_{q}$, is nearly zero for initial values of $\alpha$, and increases thereafter with $\alpha$.

The results shown in Fig. $2 \mathrm{~b}$ correspond to phase components, $\psi_{p}$ and $\psi_{q}$ calculated by using respective expressions (7b) and (8b) under similar conditions as of Fig. 2a. The phase component $\psi_{p}$ for both drive frequencies $\omega_{d}=\omega_{1 x}$ and $\omega_{d}=1.005 \omega_{1 x}$, is nearly zero for entire calculated range of $\alpha$ and related curves overlap with each other. While $\psi_{q}$, for $\omega_{d}=\omega_{1 x}$, is zero at $\alpha=0$ and abruptly goes gown to $-90^{\circ}$ as $\alpha$ increases and persists with this value for the entire calculated range of $\alpha$. On the other hand for $\omega_{d}=1.005 \omega_{1 x}$, the component $\psi_{q}$ rises steeply from $-180^{\circ}$ to $-90^{\circ}$ as $\alpha$ increases and then persists with latter value for rest of the calculated range of $\alpha$. From this it is inferred that both in-phase and quadrature signal components are at $90^{\circ}$ of phase difference.

Figure 2c reveals that for $\omega_{d}=0.95 \omega_{1 x}$, both the components $A_{p}$ and $A_{q}$, have the same nature of variation in their magnitudes as shown in Fig. 2a. However, for $\omega_{d}=1.05 \omega_{1 x}$, the magnitudes of $A_{p}$ and $A_{q}$ are almost the same as those for $\omega_{d}=0.95 \omega_{1 x}$, within the range, $\alpha<0.04 \omega_{d}$, but beyond this range of $\alpha$ both $A_{p}$ and $A_{q}$ rise steeply and peak at about $\alpha=0.06 \omega_{d}$. Thereafter, both amplitude components decrease sharply and interchange their magnitudes, thus, showing balancing natures with each other.

The significant changes have occurred in the phase characteristics for such a deviation in the drive frequency as shown in the Fig. $2 d$. For $\omega_{d}=0.95 \omega_{1 x}, \psi_{p}$ is zero and invariant with the variation of $\alpha$. At the same time, $\psi_{q}$ is about zero at $\alpha=0$ and then increases with $\alpha$ and reaches to $90^{\circ}$ which persists for the entire calculated range of $\alpha$. 
The components $\psi_{p}$ and $\psi_{q}$ for $\omega_{d}=1.05 \omega_{1 x}$, show different characteristics. The phase component $\psi_{q}$ originates at $-180^{\circ}$ and as $\alpha$ increases phase value reaches to $90^{\circ}$ at about $\alpha=.06 \omega_{d}$. Then it starts decreasing up to $-270^{\circ}$, whereas $\psi_{p}$ is zero in the beginning and then it fluctuates from zero to negative values by varying $\alpha$.
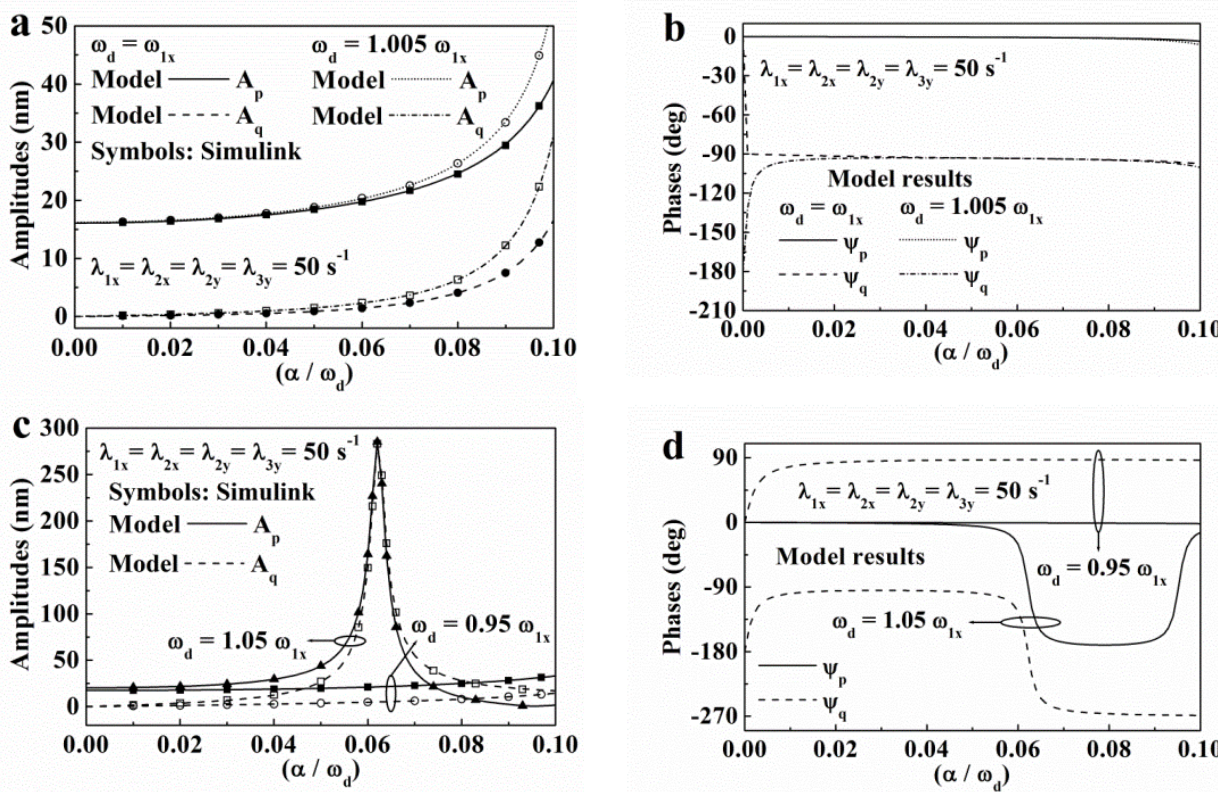

Fig. 2. Bode plots of demodulated and then filtered in-phase and quadrature components of amplitudes and corresponding phases, $\mathbf{a}$ amplitudes $\mathbf{b}$ phases for $\omega=\omega_{d}=\omega_{1 x}$ and for $\omega_{d}=1.005 \omega_{1 x}, \mathbf{c}$ amplitudes $\mathbf{d}$ phases for $\omega_{d}=1.05 \omega_{1 x}$ and for $\omega_{d}=0.95 \omega_{1 x}$, (symbols are Simulink results)

The transient response of the in-phase component with respect to time which is a result of linear acceleration is shown in Fig. 3, after demodulation and filtration. The Simulink results use the same conditions as that for the analytical analysis, which considers the settled transient response of the device. It can be observed in Fig. 3 that the device settles down at about $0.24 \mathrm{~ms}$. The time required for settling down depends on the ambient pressure at which the device is operated. The in-phase displacement component, $\bar{y}_{l p}$ is plotted using equation (7) and it clearly shows an excellent match between analytical and Simulink results. Figure 3 also shows that when the device is driven at frequency, zero-phase frequency, acceleration part is zero and gyro action is dominant, indicated by a sinusoidal variation Hence, angular rate can be measured through the in-phase output.

Figure 4 shows the transient response of the quadrature output signal $\left(\bar{y}_{l q}\right)$ calculated by using (8), which is a result of the combined action of Coriolis and Euler's forces. It is evident from the plot that the acceleration action is dominant and gyro action is zero. Hence, acceleration action can be determined from the quadrature output. This 
figure also includes Simulink data showing excellent agreement with the analytical results.
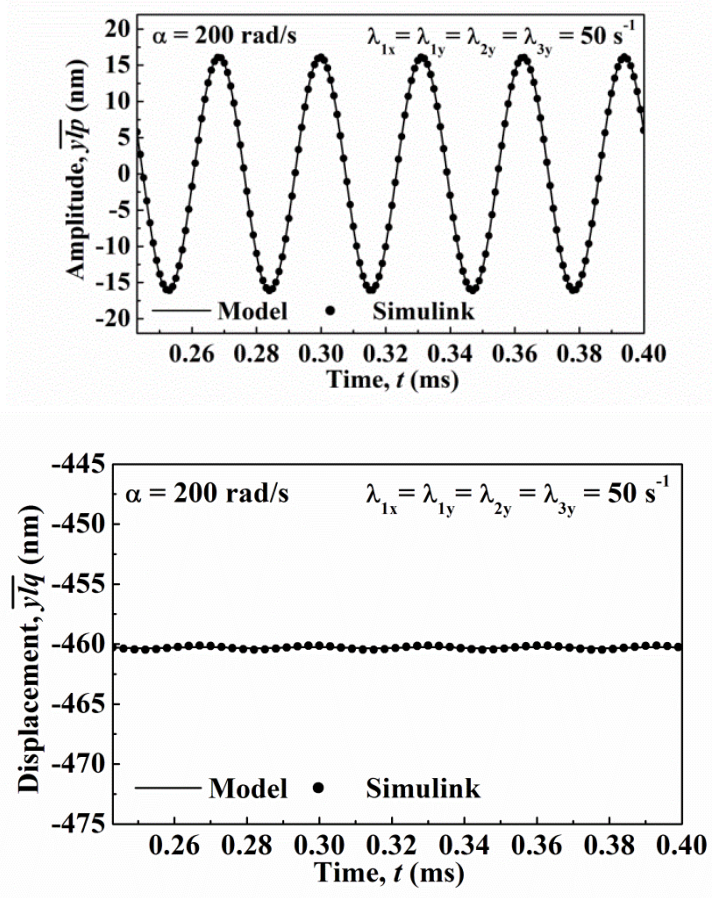

Fig. 4. Quadrature component $\left(\overline{\boldsymbol{y}}_{l q}\right)$ displacement variation with time, $\left(\mathcal{R}_{e x}=10 \mathrm{~g}\right)$

\section{Conclusion}

The simultaneous detection scheme of time varying angular rate and linear acceleration utilizes the synchronous demodulation that yields in-phase and quadrature output signals of the systems. In case of matched zero phase frequencies of both the oscillators, the associated acceleration term in in-phase component becomes ineffective and the device deliver only angular rate related signals, whereas the quadrature signal is dominant by acceleration action and that related to angular rate becomes almost insignificant. Therefore, in-phase signal can be used for acceleration detection and quadrature one for angular rate extractions. MATLAB ${ }^{\circledR} /$ Simulink model of the gyroaccelerometer system was developed in order to investigate the feasibility of such a detection scheme and simulation results have shown excellent correspondence with analytical results.

\section{References}

1. Alper SE, Akin T. Symmetrical and decoupled nickel microgyroscope on insulating substrate. Sensors and Actuators A: Physical, 2004; 115: 336-350. 
2. Acar C, Shkel A. MEMS vibratory gyroscopes-structural approaches to improve robustness, Springer, Berlin, 2009.

3. Verma P, Shekhar C, Arya SK, Gopal R. New design architecture of a 3-DOF vibratory gyroscope with robust drive operation mode and implementation. Microsystem Technologies, 2015; 21: 2175-2185.

4. Verma P, Gopal R, Butt MA, Khonina SV, Skidanov RV. Design and simulation of nonresonant 1-DOF drive mode and anchored 2-DOF sense mode gyroscope for implementation using UV-LIGA process. In: Proceedings of the SPIE 9807, 2016; DOI: $10.1117 / 12.2231372$.

5. Verma P, Juneja S, Savelyev DA, Khonina SN, Gopal R. Design and fabrication of a 1DOF drive mode and 2-DOF sense mode micro-gyroscope using SU-8 based UV-LIGA process. In: Proceedings of the AIP 1724, 2016; DOI: 10.1063/1.4945137.

6. Sung WT, Kang T, Lee JG. Controller design of a MEMS gyro-accelerometer with a single proof mass. International Journal of Control, Automation, and Systems, 2008; 6(6): 873-883.

7. Verma P, Gopal R, Arya SK. Analytical modeling and simulation of a 2-DOF drive and 1DOF sense gyro-accelerometer. Microsystem Technologies, 2013; 19(8): 1238-1249.

8. Verma P, Arya SK, Gopal R. Lumped parameter analytic modeling and behavioral simulation of a 3-DOF MEMS gyro-accelerometer. Acta Mechanica Sinica, 2015; 31(6): 910919.

9. Kazanskiy NL. Asymptotic research in computer optics. CEUR Workshop Proceedings, 2015; 1490: 151-161. DOI: 10.18287/1613-0073-2015-1490-151-161.

10. Doskolovich LL, Moiseev MA, Kazanskiy NL. On using a supporting quadric method to design diffractive optical elements. Computer Optics, 2015; 39(3): 339-346. DOI: 10.18287/0134-2452-2015-39-3-339-346.

11. Kazanskiy NL, Kharitonov SI, Khonina SN. Simulation of a hyperspectrometer based on linear spectral filters using vector Bessel beams. Computer Optics, 2014; 38(4): 770-776.

12. Kazanskiy NL, Serafimovich PG. Cloud Computing for Nanophotonic Simulations. Lecture Notes in Computer Science, 2013; 7715: 54-67. DOI: 10.1007/978-3-642-38250-5_7.

13. Golovashkin DL, Kasanskiy NL. Solving Diffractive Optics Problem using Graphics Processing Units. Optical Memory and Neural Networks (Information Optics), 2011; 20: 8589. DOI: $10.3103 / \mathrm{S} 1060992 X 11020019$.

14. Verma P, Gopal R, Arya SK. Dynamic characteristics of vibratory gyro-accelerometer. In Proceeding of the IEEE, 5th International Conference on Computers and Devices for Communication, University of Calcutta, India, 2012; DOI: 10.1109/ CODEC.2012.6509277.

15. Verma P, Agrawal P, Gopal R, Arya SK. Parametric sensitivity analysis of a 2-DOF drive and 1-DOF sense modes MEMS gyro-accelerometer structure. Advance Science Letters, 2014; 20: 1495-1498.

16. Verma P, Khan KZ, Butt MA, Khonina SN, Kazanskiy NL, Gopal R. Acceleration Characterization of Dual Purpose Gyro/Accelerometer Device using MS3110 Differential Capacitive Read Out IC. In Proceeding of IEEE, MicroCom 2016, NIT Durgapur, India.

17. Verma P, Khan KZ, Gopal R, Butt MA, Fomchenkov SA, Savelyev DA, Khonina SN, Kazanskiy NL, Skidanov RV. Analytical modeling of a non-resonant muti-DOF MEMS gyroaccelerometer. In Proceeding of the IEEE, ICECS 2016, Coimbatore, India. 